\title{
Inflammasome protein seeds plaques in Alzheimer disease
}

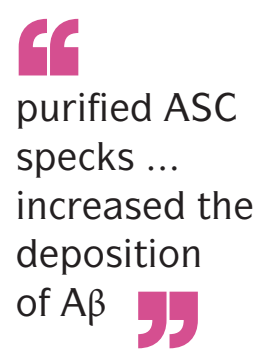

Alzheimer disease is characterized by the presence of amyloid- $\beta(A \beta)$ plaques in the brain (which are thought to cause neuronal dysfunction), as well as by neurochemical changes and inflammation. Writing in Nature, Venegas and colleagues found that aggregates of apoptosisassociated speck-like protein containing a CARD (ASC), which result from innate immune activation, can seed $A \beta$ deposition. Interfering with ASC specks reduced $A \beta$ deposition in a mouse model of Alzheimer disease.

Activation of the innate immune system is an early occurrence in numerous neurodegenerative diseases. Aberrant or misfolded proteins can be recognized by microglial



pattern recognition receptors, resulting in the activation of NOD-, LRR- and pyrin domain-containing 3 (NLRP3), a component of the inflammasome. This molecule recruits ASC, which assembles into large helical fibrils, and promotes the production and release of proinflammatory cytokines.

Although $\mathrm{A} \beta$ can activate NLRP3, innate immune activation can precede $A \beta$ production, prompting the authors to examine the contribution of NLRP 3 activation to $A \beta$ plaques. Furthermore, synthetic $\mathrm{A} \beta$ itself does not form plaques efficiently, suggesting that a cofactor is required for $A \beta$ assembly and deposition. Indeed, purified ASC specks accelerated aggregation of both of the forms of $A \beta$ that are thought to be pathogenic $\left(\mathrm{A} \beta_{1-40}\right.$ and $\mathrm{A} \beta_{1-42}$ ), suggesting that ASC specks seed $A \beta$ aggregates. This aggregation was dependent on three amino acids within the pyrin domain of ASC, which likely interact directly with $A \beta$.

In a mouse model of Alzheimer disease (APP/PS1 mice), ASC co-immunoprecipitated with $A \beta$ in brain lysates, particularly in aged mice. These proteins were not found together in the brains of wild-type mice. Immunohistochemistry confirmed this observation; even in the brains of presymptomatic mice, protein structures with an ASC core surrounded by $A \beta$ were observed. Similarly, ASC-A $\beta$ complexes were found in the brains of patients with Alzheimer disease, including those with early forms, but not in the brains of patients with other neurodegenerative diseases.

Aged APP/PS1;Asc/- mice had reduced levels of cerebral $A \beta$ and improved spatial memory compared with $A P P / P S 1$ mice. Injection of $A P P / P S 1$ brain lysates into the brains of $A P P / P S 1$ mice, but not into APP/ $P S 1 ; A s c^{-/}$mice, increased A $\beta$ deposition, suggesting a role for endogenous ASC in this process. Thus, ASC specks could seed $A \beta$ deposition, which then promotes further ASC speck formation through the activation of the innate immune system.

Reducing the interaction between ASC specks and $A \beta$ reduced $A \beta$ spreading. If $A P P / P S 1$ mice received a co-injection of an antibody specific to ASC specks along with the APP/PS1 brain homogenates, the $A \beta$ monomers and oligomers were reduced.

These results show that the NLRP3 inflammasome and its downstream activator, ASC, are key components in the development of Alzheimer disease. Targeting inflammasome components, particularly in the early stages of disease, could slow the formation of $A \beta$ plaques and thus delay the neurological sequelae in these patients.

Megan Cully

ORIGINAL ARTICLE Venegas, C. et al. Microgliaderived ASC specks cross-seed amyloid- $\beta$ in Alzheimer's disease. Nature 552, 355-361 (2017) 